\title{
Variation in working memory capacity and episodic recall: The contributions of strategic encoding and contextual retrieval
}

\author{
NASH UnSWORTh AND GREgory J. SPILlers \\ University of Georgia, Athens, Georgia
}

\begin{abstract}
The present study examined the extent to which differences in strategic encoding and contextual retrieval account for the relation between individual differences in working memory capacity (WMC) and variation in episodic recall. Participants performed a continual distractor task under either incidental- or intentional-encoding conditions. High-WMC individuals outperformed low-WMC individuals across both encoding conditions and, notably, to a greater degree in the intentional-encoding condition. These results suggest that WMC differences in episodic recall are likely due to a combination of differences in both contextual-retrieval and strategic-encoding processes. These findings are consistent with prior work showing that high-WMC individuals are better at engaging in strategic-encoding processes during the presentation of items than are low-WMC individuals and are better at using contextual cues to focus the search on correct items during retrieval.
\end{abstract}

A great deal of prior work has demonstrated that working memory capacity (WMC) is an important predictor of performance on a number of higher and lower order cognitive tasks (Engle \& Kane, 2004). Recent work has suggested that individual differences in WMC (as measured by complex span tasks) are related to differences in episodic retrieval in tasks such as free recall (Bailey, Dunlosky, \& Kane, 2008; Kane \& Engle, 2000; Unsworth, 2007). However, the reasons for this relation are still not well understood. It is possible that these differences arise primarily from differences in strategic retrieval processes, differences in strategic-encoding processes, or some combination of both. That is, variation in WMC likely reflects variation in control processes (Engle \& Kane, 2004; Unsworth \& Engle, 2007), and these control processes likely operate both at encoding, in the form of differences in encoding strategies, and at retrieval, in the form of differences in retrieval strategies and the selection of appropriate probes or cues (Atkinson \& Shiffrin, 1971).

In previous work, we (Unsworth \& Engle, 2007) have suggested that the relationship between WMC and episodic recall is due, in part, to differences in contextual-retrieval abilities. In line with Glenberg and colleagues (Glenberg et al., 1980; Glenberg \& Swanson, 1986), we suggested that, at encoding, items are associated with multiple levels of context (e.g., global, list-specific, and word-specific contexts). At retrieval, these contextual features are used as cues/probes to constrain the search to the items from the most recently presented list. In terms of individual differences in WMC, we suggested that low-WMC individuals are less efficient than high-WMC individuals at selecting appropriate retrieval strategies and retrieval cues/probes (particularly, contextual cues), which leads to the inclusion of more irrelevant items in their search sets. The inclusion of more irrelevant items in the search sets of low-WMC individuals increases the overall size of their search sets (cue overload), leading to a less accurate and slower search.

According to this contextual-retrieval hypothesis, lowWMC individuals demonstrate poorer episodic recall than do high-WMC individuals because they use less efficient contextual cues to effectively constrain the search to only the relevant items. Evidence consistent with this hypothesis has come from studies in which high- and low-WMC differences in free recall tasks have been examined, where low-WMC individuals not only recalled fewer target items than did high-WMC individuals, but also were slower to recall items and recalled more intrusions than did highWMC individuals (Unsworth, 2007). Collectively, these results suggest that low-WMC individuals search through a larger set of items than do high-WMC individuals, due to the fact that low-WMC individuals are less able to use contextual cues to focus the search. Furthermore, simulations that tested various explanations for differences between high- and low-WMC individuals in episodic recall (e.g., contextual retrieval, amount of resources, strength of encoding, and processing speed) showed that only the contextual-retrieval account of the data was consistent with the overall pattern of results (Unsworth, 2007). Thus, we suggested that the primary reason for high- and lowWMC differences in episodic recall was differences in the ability to use contextual cues at retrieval, and not basic differences in encoding abilities.

However, other work has suggested that WMC differences in episodic recall are partially due to differences in

N. Unsworth, nunswor@uga.edu 
strategic-encoding abilities between high- and low-WMC individuals (Bailey et al., 2008; see also Cokely, Kelley, \& Gilchrist, 2006; Dunlosky \& Kane, 2007; Turley-Ames \& Whitfield, 2003). Specifically, Bailey et al. found that participants used a variety of strategies (rehearsal, imagery, grouping, etc.) on both complex span tasks and measures of free and cued recall. Furthermore, Bailey et al. found that strategy use partially mediated the relation between WMC and recall. Overall, this work suggests that differential encoding strategies partially account for WMC differences in episodic recall. Thus, it remains an open question as to whether WMC differences in episodic recall are best thought of as differences in contextual-retrieval abilities, strategic-encoding abilities, or both.

\section{The Present Study}

The purpose of the present study was to examine the extent to which contextual retrieval, strategic encoding, or both account for WMC differences in episodic recall. In order to address this issue, high- and low-WMC individuals performed a continual distractor task under either incidental- or intentional-encoding conditions on the first list. An incidental-encoding condition was used to minimize the use of encoding strategies (rehearsal) by the participants (Glenberg et al., 1980; Marshall \& Werder, 1972). That is, when there is no expectation of a later recall test, there is no reason for participants to actively try to encode the words via the use of strategies (such as rehearsal). Accordingly, with incidental encoding, at retrieval participants should rely on contextual cues present during the recall period, in an attempt to reinstate the encoding context and retrieve the words from the list, leading to strong recency effects but diminished primacy effects (Glenberg et al., 1980; Marshall \& Werder, 1972). Furthermore, because participants should rely on contextual cues at retrieval in the incidental condition, their first recall should be a recency item. With intentional encoding, participants should rely on encoding strategies (rehearsal) to remember the words, leading to strong primacy effects, and participants should start their recall with a primacy item. Thus, differences between incidental and intentional encoding should lead to differences in the serial position functions for proportion correct and probability of first recall.

In terms of $\mathrm{WMC}$, if differences in episodic recall are solely due to contextual retrieval, high-WMC individuals should recall more items than should low-WMC individuals in both incidental- and intentional-encoding conditions, and WMC should not interact with encoding condition, indicating that the magnitude of the effect was the same in both conditions. If WMC differences in episodic recall are due to differences in strategic encoding, high- and low-WMC individuals should not differ in recall in the incidental-encoding condition but should differ in the intentional-encoding condition. Finally, if WMC differences in recall are due to both processes, high- and low-WMC differences should arise in both encoding conditions, and the magnitude of the effect should increase in the intentional-encoding condition, in which both processes contribute to overall performance. Furthermore, in line with Bailey et al. (2008), if WMC differences are partially due to differences in strategies, high- and low-WMC individuals should differ in the strategies that they report using. To examine this, after the first list, all the participants performed two additional intentional-encoding trials and, after the trials, were asked to report the strategies that they had used to remember the items (Bailey et al., 2008). This should allow for an assessment of specific strategies and possible WMC differences in strategies on the current task. This examination of encoding and retrieval processes should help shed light on the nature of WMC differences overall and the reason why WMC seems to be related to episodic recall more broadly.

\section{METHOD}

\section{Participants and WMC Screening}

The participants were recruited from the participant pool at the University of Georgia. Individuals were selected on the basis of a $z$ score composite of the three complex span tasks. Only participants falling in the upper (high-WMC individuals) and lower (low-WMC individuals) quartiles of the composite distribution were selected.

Operation span. The participants solved a series of math operations while trying to remember a set of unrelated letters that were presented for $1 \mathrm{sec}$ each. Immediately after the letter had been presented, the next operation was presented. Three trials of each list length (three to seven) were presented, with the order of list length varying randomly. At recall, letters from the current set were recalled in the correct order by clicking on the appropriate letters. The participants received three sets (with a list length of two) of practice. For all of the span measures, the score was the proportion of correct items in the correct position.

Symmetry span. In this task, the participants were required to recall sequences of red squares within a matrix while performing a symmetry judgment task. In the symmetry judgment task, the participants were shown an $8 \times 8$ matrix with some squares filled in black. The participants decided whether the design was symmetrical about its vertical axis. The pattern was symmetrical half of the time. Immediately after determining whether the pattern was symmetrical, the participants were presented with a $4 \times 4$ matrix with one of the cells filled in red for $650 \mathrm{msec}$. At recall, the participants recalled the sequence of red square locations in the preceding displays, in the order in which they had appeared, by clicking on the cells of an empty matrix. There were three trials of each list length, with list length ranging from two to five. The same scoring procedure as that for Ospan was used.

Reading span. The participants were required to read sentences while trying to remember the same set of unrelated letters as that for Ospan. For this task, the participants read a sentence and determined whether the sentence made sense or not (e.g., "The prosecutor's dish was lost because it was not based on fact. ?"). Half of the sentences made sense, whereas the other half did not. Nonsense sentences were made by simply changing one word (e.g., "dish" from "case") from an otherwise normal sentence. After the participants indicated whether the sentence made sense or not, they were presented with a letter for $1 \mathrm{sec}$. At recall, letters from the current set were recalled in the correct order by clicking on the appropriate letters. There were three trials of each list length, with list length ranging from three to seven. The same scoring procedure as that for Ospan was used.

\section{Composite Score}

For the composite score, the scores for the three complex span tasks were $z$-transformed for each participant. These $z$ scores were then averaged together, and quartiles were computed from the averaged distribution. The participants were 52 (26 in each encoding condition) high-WMC individuals $(z-\mathrm{WMC}=0.75, S D=0.22)$ 


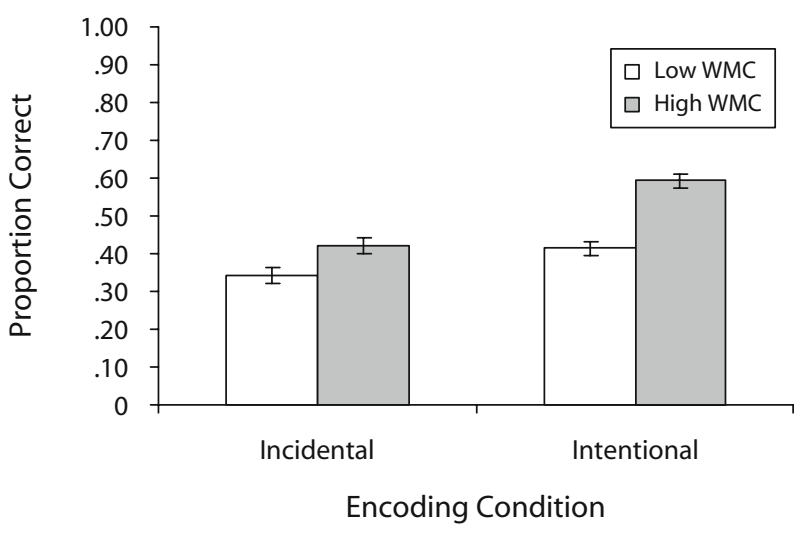

Figure 1. Proportion correct as a function of encoding condition (incidental vs. intentional) and working memory capacity (WMC; high vs. low). Error bars represent one standard error of the mean.

and 52 (26 in each encoding condition) low-WMC individuals $(z$-WMC $=-1.16, S D=0.66)$, as determined by the composite measure. The mean age for both groups was 18.9 years, which did not differ as a function of WMC $(p>.65)$. Both groups were composed of $67 \%$ females.

\section{Continual Distractor Free Recall}

On List 1 , the participants were given a list of 10 common nouns. The participants were told that they would be performing an attention-switching task in which they switched between arranging digits in descending order and making living/nonliving judgments on words. Specifically, before and after each word presentation, the participants were required to arrange four separate three-digit numbers (presented for $2 \mathrm{sec}$ each) in descending order on a sheet of paper. Next, a word was presented for $2.5 \mathrm{sec}$, and the participants had to make a living or nonliving judgment by pressing the "F" or "J" key, respectively. This sequence was repeated for all 10 words. Following presentation of the last word, the participants engaged in an additional 30 -sec distractor activity (e.g., 15 three-digit numbers instead of 4) before recall. After the distractor task, the participants were given a recall test in which they saw "???" and were told to type in as many of the words from the attention-switching task as possible. The participants had $45 \mathrm{sec}$ for recall. Half of the participants were told that they were performing only the attention-switching task, and there was no indication that they would have to remember the words (incidental encoding). The other half of the participants were told that they were performing the attention-switching task but that they should also try to remember the words for a later test (intentional encoding). Following the first list, all the participants were told that they would be performing the task on an additional two lists and that they should try to remember the words for later test (intentional encoding). Otherwise, the procedure for Lists 2 and 3 was identical to that for List 1. Immediately following Lists 2 and 3 , the participants reported which strategies (if any) they had used on the intentional lists. Strategies included reading each word as it appeared, repeating the words as much as possible, using sentences to link the words, using mental imagery, grouping the words in a meaningful way, or utilizing some other strategy. The participants could indicate that they had used more than one strategy.

\section{RESULTS}

First, proportion correct on List 1 was examined with a 2 (encoding condition: incidental vs. intentional) $\times$ 2 (WMC: high vs. low) $\times 3$ (serial position: primacy, midlist, or recency) mixed ANOVA with encoding condition and $\mathrm{WMC}$ as the between-subjects factors. ${ }^{1}$ The ANOVA yielded a main effect of encoding condition $\left[F(1,100)=33.12, M S_{\mathrm{e}}=.06, p<.01, \eta_{\mathrm{p}}^{2}=.19\right]$, in which more items were recalled in the intentional condition $(M=.51, S E=.02)$ than in the incidental condition $(M=.38, S E=.01)$. There was also a marginal main effect of serial position $\left[F(2,200)=2.84, M S_{\mathrm{e}}=.09, p<\right.$ $\left..07, \eta_{\mathrm{p}}^{2}=.06\right]$, in which primacy and recency items tended to be better recalled than midlist items. Furthermore, there was a main effect of WMC $\left[F(1,100)=20.87, M S_{\mathrm{e}}=\right.$ $\left..06, p<.01, \eta_{\mathrm{p}}^{2}=.17\right]$, in which high-WMC individuals recalled more items overall $(M=.50, S E=.02)$ than did low-WMC individuals $(M=.38, S E=.02)$.

As is shown in Figure 1, there was also a significant encoding condition $\times$ WMC interaction $[F(1,100)=3.92$, $\left.M S_{\mathrm{e}}=.06, p<.05, \eta_{\mathrm{p}}^{2}=.04\right]$, such that the difference between high- and low-WMC individuals was greater in

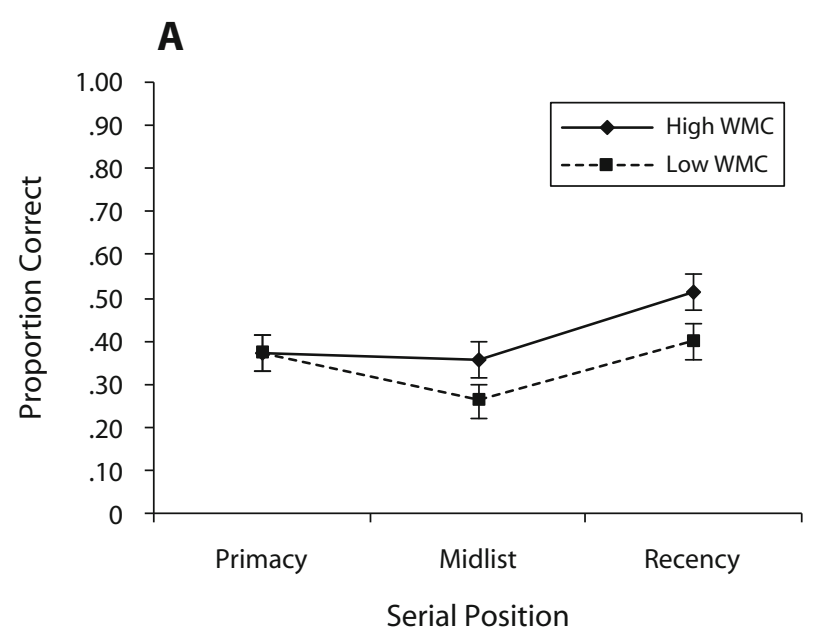

B

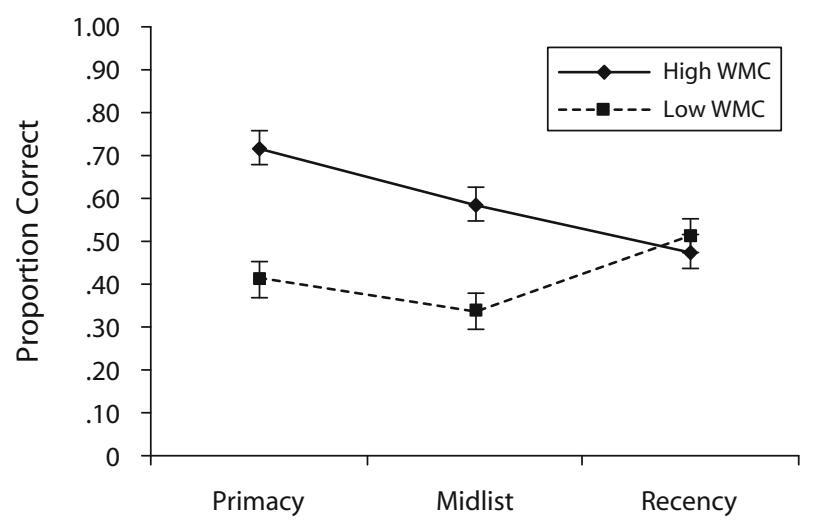

Serial Position

Figure 2. Proportion correct as a function of serial position (primacy, midlist, or recency) and working memory capacity (WMC; high vs. low) for each encoding condition separately (panel A, incidental encoding; panel $B$, intentional encoding). Error bars represent one standard error of the mean. 


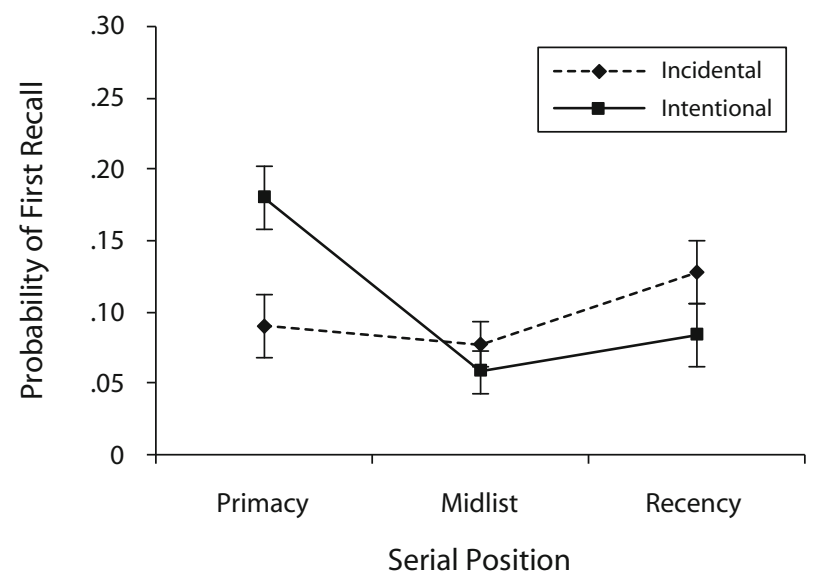

Figure 3. Probability of first recall as a function of serial position (primacy, midlist, or recency) and encoding condition (incidental vs. intentional). Error bars represent one standard error of the mean.

the intentional than in the incidental condition. Critically, however, high- and low-WMC individuals differed in both conditions, with high-WMC individuals recalling more items than did low-WMC individuals (both $p$ s $<.05$ ). Thus, WMC differences were apparent in both conditions, but the difference was greater in magnitude in the intentional condition. Furthermore, as is shown in Figure 2, the three-way interaction was significant $[F(2,200)=3.91$, $\left.M S_{\mathrm{e}}=.09, p<.05, \eta_{\mathrm{p}}^{2}=.04\right]$, suggesting that high- and low-WMC individuals differed in midlist and recency portions of the serial position curve under incidental encoding but differed primarily in primacy and midlist portions of the curve under intentional encoding. Thus, high-, but not low-, WMC individuals primarily recalled recency items under incidental encoding and then switched to primarily recalling primacy items under intentional encoding.

Next, probability of first recall (PFR) was examined with a 2 (encoding condition) $\times 2($ WMC) $\times 3$ (serial position) mixed ANOVA with encoding condition and WMC as the between-subjects factors. PFR refers to the number of times the first word recalled comes from a given serial position, divided by the number of times the first recalled word could have come from that serial position. The ANOVA yielded a main effect of serial position $\left[F(2,200)=3.78, M S_{\mathrm{e}}=.03, p<.05, \eta_{\mathrm{p}}^{2}=.04\right]$, in which PFR was greatest for primacy and recency items. Importantly, as in shown in Figure 3, this effect was quali- fied by a significant encoding condition $\times$ serial position interaction $\left[F(2,200)=4.23, M S_{\mathrm{e}}=.03, p<.05, \eta_{\mathrm{p}}^{2}=\right.$ $.04]$, in which PFR was generally larger for recency items in the incidental condition but was greatest for primacy items in the intentional condition. None of the other effects reached conventional levels of significance (all $p \mathrm{~s}>.10)$. Thus, under incidental-encoding conditions, the participants generally started their recall with recency items, whereas under intentional-encoding conditions, they started their recall with primacy items, suggesting that the participants' order-of-report strategies changed as a function of encoding condition. ${ }^{2}$

We next examined recall performance on Lists 2 and 3 and differences in reported strategies for all the participants, regardless of encoding condition on the first list. These analyses should illuminate WMC differences under intentional-encoding conditions. Note that there was no difference between Lists 2 and 3 ( $p>.65$ ), so they were combined for all the analyses. Shown in Table 1 are the proportions of high- and low-WMC individuals who reported using one of the six strategies. As can be seen, most of the participants reported reading the words as they appeared and repetition as the strategy that they used, and fewer participants reported using more elaborate encoding strategies, such as grouping or imagery. In terms of WMC differences in strategy use, the only significant effect was a difference in the repetition strategy, with more high- than low-WMC individuals reporting that they had used that strategy $[t(102)=2.56, p<.01$; all other $p \mathrm{~s}>.15]$. Thus, on the intentional lists, high-WMC individuals reported using repetition more so than did low-WMC individuals. Given that WMC differences were found for Lists 2 and 3 combined $\left[F(1,100)=28.16, M S_{\mathrm{e}}=.02, p<.01, \eta_{\mathrm{p}}^{2}=\right.$ $.22]$, with high-WMC individuals $(M=.69, S E=.02)$ recalling more items than did low-WMC individuals $(M=$ $.54, S E=.02)$, we next examined whether this effect would be reduced once differences in using the repetition strategy were covaried out. That is, if WMC differences on the intentional lists are partially due to differences in strategy use (here, repetition), covarying out differences in strategy use should reduce the effect of span. As was expected, the effect of span was reduced once strategy use was covaried out $\left[F(1,96)=11.14, M S_{\mathrm{e}}=.03, p<.01\right.$, $\left.\eta_{\mathrm{p}}^{2}=.11\right]$. In particular, note that $\eta_{\mathrm{p}}^{2}$ dropped from .22 to .11 , and this difference was significant $[F(4,100)=3.85$, $p<.01]$. Thus, part of the difference between high- and low-WMC individuals in intentional recall was due to differences in strategy use (see note 2).

Table 1

Proportions of Reported Strategy Use As a Function of Strategy and Working Memory Capacity (WMC)

\begin{tabular}{|c|c|c|c|c|c|c|c|c|c|c|c|c|}
\hline \multirow[b]{3}{*}{ WMC } & \multicolumn{12}{|c|}{ Strategy } \\
\hline & \multicolumn{2}{|c|}{ Read } & \multicolumn{2}{|c|}{ Repetition } & \multicolumn{2}{|c|}{ Imagery } & \multicolumn{2}{|c|}{ Sentence } & \multicolumn{2}{|c|}{ Grouping } & \multicolumn{2}{|c|}{ Other } \\
\hline & $M$ & $S E$ & $M$ & $S E$ & $M$ & $S E$ & $M$ & $S E$ & $M$ & $S E$ & $M$ & $S E$ \\
\hline High & .92 & .04 & .79 & .06 & .15 & .05 & .08 & .04 & .19 & .06 & .12 & .05 \\
\hline Low & .85 & .05 & .56 & .07 & .27 & .06 & .04 & .03 & .17 & .05 & .13 & .05 \\
\hline
\end{tabular}

Note-Proportions of strategies sum to greater than 1.0 because the participants were allowed to report using more than one strategy. 


\section{DISCUSSION}

The present study examined the extent to which WMC differences on free recall tasks are due to differences in strategic encoding and contextual retrieval. Participants performed a continual distractor task under both incidental- and intentional-learning conditions. Consistent with prior research (e.g., Glenberg et al., 1980; Marshall \& Werder, 1972), intentional encoding resulted in better overall performance than did incidental encoding. Furthermore, there was a substantial primacy effect present in the intentional-encoding condition, and the participants typically started their recall with a primacy item. In the incidental condition, however, the primacy effect was reduced, whereas the recency effect was strengthened and the participants typically began recall with a recency item. These results are consistent with the idea that in the incidental-encoding condition, the participants relied primarily on contextual retrieval, since they likely did not use intentional-encoding strategies (i.e., rehearsal) to encode the items. In the intentional condition, however, the participants engaged in rehearsal during encoding, leading to a strong primacy effect, in addition to relying on contextual-retrieval processes.

In terms of WMC differences, high- and low-WMC individuals differed in their recall performance in both incidental- and intentional-encoding conditions, suggesting that recall differences between high- and low-WMC individuals were partially due to contextual-retrieval processes and were not due solely to strategic-encoding differences (Unsworth, 2007). That is, given that highWMC individuals outperformed low-WMC individuals in the incidental condition, it is clear that differences in strategic encoding, per se, cannot fully account for WMC differences in free recall. At the same time, the significant interaction between WMC and encoding condition suggested that the difference between high- and low-WMC individuals in free recall increased under intentional encoding and that this was primarily due to an increase in primacy for the high-WMC individuals. That is, under intentional-encoding conditions, high-WMC individuals likely relied on better encoding strategies (Bailey et al., 2008) during list presentation and more efficient contextual cues during retrieval than did low-WMC individuals. Indeed, an examination of strategy use on the intentional lists (Lists 2 and 3) suggested that high-WMC individuals were more likely than low-WMC individuals to rehearse the words during encoding. Furthermore, these differences in strategy use partially accounted for WMC differences in recall on the intentional lists. Thus, consistent with Bailey et al., differences in strategic encoding accounted for shared variance between WMC and recall, but they did not account for all of the variance.

The results of the present study suggest that WMC differences in free recall are likely due to differences in both contextual-retrieval (Unsworth, 2007) and strategic-encoding (Bailey et al., 2008) processes. High-WMC individuals are better at engaging in strategic-encoding processes during the presentation of items than are low-WMC individuals and are better at using contextual cues/probes to focus the search on correct items during retrieval. Specifying only encoding processes or only retrieval processes as the primary mechanism underlying differences in $\mathrm{WMC}$ and recall cannot fully account for prior work or the present results.

It should be noted that it is also possible that the differences found in the incidental condition represent inherent encoding differences between high- and low-WMC individuals, whereby high-WMC individuals simply encode information better than do low-WMC individuals, regardless of intentional-encoding strategies. These inherent encoding differences might include differences in the ability to bind item information to contextual features, which would then lead to the use of better contextual cues at retrieval. Our present results cannot necessarily speak to these inherent encoding differences, given that we were concerned primarily with differences in strategicencoding processes.

Furthermore, a probable explanation is that differences between high- and low-WMC individuals are due to differences in the ability to reinstate the encoding context at retrieval (Unsworth \& Engle, 2007), similar to encoding specificity (Tulving \& Thomson, 1973). That is, highWMC individuals may be better than low-WMC individuals at encoding information as distinct, which then would result in a more specific cue/probe at retrieval, leading to a more focused search set. Consistent with the present results, this suggests that high- and low-WMC individuals not only differ in encoding and retrieval, but also differ in the ability to reinstate the encoding context at retrieval. Future work should be devoted to examining differences in both encoding and retrieval, as well as WMC differences in encoding retrieval interactions (i.e., encoding specificity). Examining WMC differences in encoding and retrieval processes should help elucidate the relation between WMC and recall and should lead to a better understanding of the nature of WMC differences and their relation to higher order cognitive processes, such as intelligence.

\section{AUTHOR NOTE}

Thanks to Gene Brewer, Arthur Glenberg, and an anonymous reviewer for comments on an earlier version of the manuscript. Thanks to Heather Bailey for providing us with the strategy report materials. Correspondence concerning this article should be sent to N. Unsworth, Department of Psychology, University of Georgia, Athens, GA 30602-3013 (e-mail: nunswor@uga.edu).

\section{REFERENCES}

Atkinson, R. C., \& Shiffrin, R. M. (1971). The control of short-term memory. Scientific American, 225, 82-90.

Bailey, H., Dunlosky, J., \& Kane, M. J. (2008). Why does working memory span predict complex cognition? Testing the strategy affordance hypothesis. Memory \& Cognition, 36, 1383-1390.

Cokely, E. T., Kelley, C. M., \& Gilchrist, A. L. (2006). Sources of individual differences in working memory: Contributions of strategy to capacity. Psychonomic Bulletin \& Review, 13, 991-997.

Dunlosky, J., \& Kane, M. J. (2007). The contributions of strategy use to working memory span: A comparison of strategy assessment methods. Quarterly Journal of Experimental Psychology, 60, 1227-1245.

Engle, R. W., \& Kane, M. J. (2004). Executive attention, working memory capacity, and a two-factor theory of cognitive control. In B. H. Ross (Ed.), The psychology of learning and motivation (Vol. 44, pp. 145-199). New York: Elsevier.

Glenberg, A. M., Bradley, M. M., Stevenson, J. A., Kraus, T. A., 
TkachuK, M. J., Gretz, A. L., ET AL. (1980). A two-process account of long-term serial position effects. Journal of Experimental Psychology: Human Learning \& Memory, 6, 355-369.

Glenberg, A. M., \& Swanson, N. G. (1986). A temporal distinctiveness theory of recency and modality effects. Journal of Experimental Psychology: Learning, Memory, \& Cognition, 12, 3-15.

KAnE, M. J., \& ENGLE, R. W. (2000). Working memory capacity, proactive interference, and divided attention: Limits on long-term retrieval. Journal of Experimental Psychology: Learning, Memory, \& Cognition, 26, 333-358.

Marshall, P. H., \& Werder, P. R. (1972). The effects of the elimination of rehearsal on primacy and recency. Journal of Verbal Learning \& Verbal Behavior, 11, 649-653.

Tulving, E., \& Thomson, D. (1973). Encoding specificity and retrieval processes in episodic memory. Psychological Review, 80, 352-372.

Turley-Ames, K. J., \& WhitFiEld, M. M. (2003). Strategy training and working memory task performance. Journal of Memory \& Language, 49, 446-468.

UNSWORTH, N. (2007). Individual differences in working memory capacity and episodic retrieval: Examining the dynamics of delayed and continuous distractor free recall. Journal of Experimental Psychology: Learning, Memory, \& Cognition, 33, 1020-1034.

UnSWORTH, N., \& ENGLE, R. W. (2007). The nature of individual differences in working memory capacity: Active maintenance in primary memory and controlled search from secondary memory. Psychological Review, 114, 104-132.

\section{NOTES}

1. Primacy items were Positions 1-3, midlist items were Positions 4-7, and recency items were Positions 8-10.

2 . Note that, in a prior version of this experiment, we had a separate sample of high- $(n=32)$ and low- $(n=34) \mathrm{WMC}$ individuals perform the incidental condition for List 1 and then perform Lists 2 and 3 under intentional-learning conditions. Thus, encoding condition was manipulated within subjects. The results for this experiment were exactly the same as those reported for the between-subjects experiment reported in the present study. Specifically, high- and low-WMC individuals differed under incidental-encoding conditions, and this difference increased under intentional-encoding conditions $\left[F(1,64)=5.99, M S_{\mathrm{e}}=.19\right.$, $\left.p<.05, \eta_{\mathrm{p}}^{2}=.09\right]$. Furthermore, consistent with the present study, participants were more likely to start their recall with a recency item in the incidental-encoding condition and were more likely to start their recall with a primacy item in the intentional-encoding condition $[F(9,576)=$ $\left.11.36, M S_{\mathrm{e}}=.06, p<.01, \eta_{\mathrm{p}}^{2}=.15\right]$.

(Manuscript received April 3, 2009; revision accepted for publication November 16, 2009.) 